\title{
Subjective effects of antipsychotic drugs and their relevance for compliance and remission
}

\author{
DIETER NABER
}

\begin{abstract}
Only recently, success criteria became more ambitious and include a more thorough consideration of negative symptoms and cognitive dysfunction. The most important change within the last decade is the long overdue consideration of the patient's perspective. His/her subjective well-being, often unchanged or even worsened by typical antipsychotics, was neglected for a long time. One reason was the prejudice that schizophrenic patients are not able to self-rate their quality of life. Another reason was the belief that such data are not necessary because the psychiatrists' perspective, "objective" psychopathology, includes these domains. Among other scales, a self-report instrument has been constructed to evaluate "subjective well-being under neuroleptics" (SWN). This scale was used in numerous open and controlled trials, indicating: a) patients, if no longer acutely psychotic or suffering from severe cognitive deficits, are able to reliably assess their subjective well-being, b) high SWN is correlated with high compliance, c) atypical antipsychotics increase SWN, and d) individual improvements of SWN and of PANSS are not strongly related. Moreover, several studies found that early improvement of subjective well-being is a major predictor for the chance of remission. All these data indicate that a better consideration of the patient's perspective is possible and necessary.
\end{abstract}

Regarding the relevance of antipsychotic drugs in the treatment of schizophrenia, it is surprising that a thorough evaluation of the patients' subjective experience has received so little scientific attention, at least during the period of typical antipsychotics. The numerous complaints from patients with schizophrenia about adverse subjective effects such as affective blunting, cognitive slowing as well as loss of spontaneity are well known since the beginning of antipsychotic treatment, they have been described by a variety of terms such as neuroleptic dysphoria, pharmacogenic depression, akinetic depression, neuroleptic depression or neuroleptic-induced anhedonia. Very often, it was assumed that these emotional side effects were only of clinical relevance if motor symptoms were obvious. Among the pioneers recognising the importance of the patients' perspective were van Putten et al. (1981), who revealed the significant relationship between subjective experience and compliance as well as Awad whose group developed the first selfreport to predict medication compliance (Hogan et al.,

Address for correspondence: Professor D. Naber, University Medical Center Hamburg-Eppendorf, Department of Psychiatry, Martinistrasse 52, 20246 Hamburg (Germany).

Fax: +49-40-42803.2999

E-mail: naber@uke.uni-hamburg.de

Declaration of Interest: Dieter Naber received research grants and speaker's fee from and is on the Advisory of AstraZeneca, Eli Lilly, Janssen-Cilag, Lundbeck, Organon, Servier and Wyeth.
1983). For 20-30 years after the introduction of antipsychotic treatment, most research on side effects and reasons for non-medication adherence focused on motor symptoms. The more subtle emotional restrictions, crosssectionally difficult to differentiate from primary or secondary negative symptoms, were excluded from contemporary research.

There are probably several reasons, which might explain why the patients' perspective was neglected over such a long period (Windgassen, 1992; Naber, 2005): Many psychiatrists believed or were convinced that their schizophrenic patients are not able to reliably assess their subjective well-being. Their complaints were attributed to the illness and they were "only" subjective. This ignoring is particularly disconcerting in psychiatry, a field where many diagnoses are based on patients' statements, not objectively verifiable. The subjective suffering from hallucinations or delusions is hardly ever doubted, but patients' opinion regarding the quality of pharmacological treatment was considered as not qualified. However, over the last years, numerous studies have shown that patients with schizophrenia, if no longer acutely psychotic and not suffering from severe cognitive impairment, are able to self-rate their affective state, subjective wellbeing or quality of life in a reliable manner (a.o. Naber et al., 2001; Voruganti et al., 1998). In the era of typical antipsychotics, psychiatrists' possibilities to respond to the complaints of their patients were rather limited. The reduction of neuroleptic dosage or better information about the necessity, benefits and risks of treatment were 
not possible or not successful and, because of the pharmacological and clinical similarities, the switch from one high-potent typical antipsychotic to another one most often proved not to be useful. The potentially helpful switch from a high-potent to a low-potent antipsychotic (or vice-versa) was seldom done. A major reason for the late scientific interest in the patients' perspective was probably the belief of many psychiatrists that they know their patients sufficiently well and do not need additional systematic information on their subjective experience. Again, several trials revealed that both perspectives differ markedly regarding efficacy and tolerability of antipsychotic treatment. A good example is the study by Voruganti et al. (2000), who found in a double-blind comparison between typical and atypical antipsychotics that many of the advantages of the atypicals, particularly the improvement in quality of life, were detected "only" by patients' self-reports, but were missed by psychiatrists' measurements.

The development of atypical antipsychotics with none or less motor symptoms as well as less emotional and affective restrictions led not only to the end of a psychopharmacological dogma (no antipsychotic efficacy without typical motor side effects) but also to more ambitious success criteria, including the patients' perspective, their subjective well-being and/or quality of life (Karow $\&$ Naber, 2002). Finally, about 30 years after the beginning of antipsychotic treatment, an open clozapine trial was the first study in which the effect of antipsychotic treatment on quality of life was measured (Meltzer et al., 1990). Measurement of antipsychotics' subjective effects is certainly confounded by symptoms, attitudes towards medication or other non-pharmacological factors. Neither patients nor psychiatrists are able to differentiate these different components of subjective well-being under neuroleptic treatment, that is why de Haan et al. (2002) proposed to operationalize "subjective experience, as constituting aspects of mental or physical state, which patients report regardless of etiological attributions." Most definitions agree that subjective well-being is a major component of quality of life, which is the more comprehensive outcome parameter, often measured by expert-rated and/or generic scales. The most popular one is the "Quality of Life" scale (Heinrichs et al., 1984), which includes items such as housing, money and number of credit cards, not very sensitive to treatment effects.

Among other reasons, the impressive reports of patients switched from typical antipsychotics to clozapine stimulated the author to systematically investigate the patients' perspective and to develop the "Subjective Well-being under Neuroleptic treatment" scale (SWN), a 20-item Likert scale with 5 subscores, 10 positive and 10 negative items. It has been translated into 15 languages, its psychometric properties have been replicated by a.o. Dutch and Italian authors (de Haan et al., 2002; Balestrieri et al., 2006). Several trials have shown that SWN is strongly related to compliance (Karow et al., 2007; Naber, 1995), and that it is sensitive to effects of antipsychotic treatment. A significant and clinically relevant improvement of SWN occurred within 2-6 weeks, atypical antipsychotics were found to be superior compared to typical antipsychotics (Naber, 1995; Lambert et al., 2006). The first double-blind trial, where SWN was the primary outcome criterion, was the 26-week comparison between olanzapine and clozapine (Naber et al., 2005). There was no significant difference, both drugs induced a marked improvement of SWN within 2 to 4 weeks. Similar as in a previous study (Naber et al., 2001), again the individual improvements of psychopathology and of SWN were not strongly related $(r=-0.3--0.4)$. These studies and others strongly agree in two very relevant issues: 1) self-ratings by schizophrenic patients are possible and useful (patients are able to fill out a scale in a useful way) and 2), they are necessary (the patients' and the psychiatrists' perspectives differ markedly)!

Regarding the etiology of antipsychotics' subjective effects, recent human brain imaging studies showed significant relationships between dopamine blockade and reduced subjective well-being (de Haan et al., 2000) as well as dysphoria induced by dopamine depletion in the nigrostriatal area (Voruganti \& Awad, 2004). These results are in line with animal data, which demonstrated that (at least some) atypicals inhibit the reward systems less than typicals. This might be the neurobiological explanation why most patients with schizophrenia markedly prefer the atypicals. This hypothesis was strongly supported by a PET-study (Mizrahi et al., 2007) with the highly significant and rather impressive correlation between dopamine D2 receptor blockade and the SWN. In the striatum, it was found to be $r=-0.66$, in the temporal lobe it was even higher, $r=-0.76$.

Moreover, recent studies indicate the relevance of early improvement of subjective well-being regarding remission and long-term prognosis. In a short-term trial of 12 weeks in 727 patients treated with amisulpride (Lambert et al., 2007), early improvement of SWN (within 4 weeks) was the most important predictor for improvement of psychopathology and of social functioning in the following 8 weeks. Very similar are the data of a 3-year trial (Lambert et al., 2006) in 2,960 patients. Again, early improvement of SWN was a major predictor to reach symptomatic and functional remission. These 
data indicate that patients with no improvement of SWN in the first weeks need to be identified early with subsequent treatment adaptation such as verification of compliance, change of antipsychotic drug or dosage, intensification of psychosocial interventions.

Regarding the impact of side effects on patients' subjective well-being, antipsychotic treatment (typical or atypical) is of relevance. In patients, treated with typical antipsychotics, SWN is mostly reduced by motor symptoms while in those treated with atypical antipsychotics, SWN is mostly correlated with negative symptoms (Putzhammer et al., 2005). If they suffer from side effects, intraindividually very heterogeneous, sedation, weight gain and sexual dysfunction are most relevant.

Since the Quality of Life scale by Heinrichs et al. (1984) is still the one most often used, recent data on the correlation between QLS and SWN are of interest. In a 12-month trial in 1,462 patients, the relationship was $\mathrm{r}=$ 0.49 at baseline, 0.62 at 3 months, 0.66 at 6 months, 0.66 at 9 months and 0.78 at 12 months (Wehmeier et al., 2007a). Moreover, these authors found some interesting factors explaining some of the differences between patient- and expert-rated quality of life (Wehmeier et al., 2007b).

Current improved possibilities in individualizing antipsychotic and psychosocial treatment of schizophrenia as well as patients' wish for more autonomy and greater participation in treatment decisions are strong reasons to thoroughly and systematically evaluate patients' experiences. The heterogeneity of atypical antipsychotics with marked differences in their side effect profile increases the difficulty, but also the chance to select an effective and tolerable drug for the individual patient. A better consideration of the patients' perspective might improve therapeutic alliance, medication adherence and the chance to achieve remission.

\section{REFERENCES}

Balestrieri M., Giaroli G., Mazzi M. \& Bellantuono C. (2006). Performance of the Italian Version of the Subjective Well-being under Neuroleptic (SWN) Scale in Schizophrenic Outpatients. Pharmacopsychiatry 39, 81-84.

De Haan L., Lavalaye J., Linszen D., Dingemans P.M. \& Booje J. (2000). Subjective experiences and striatal dopamine D2 receptor occupancy in patients with recent onset schizophrenia treated with olanzapine or risperidone. American Journal of Psychiatry 157, 1019-1020.

De Haan L., Weisfelt M., Dingemans P.M., Linszen D.H. \& Wouters L. (2002). Psychometric properties of the subjective well-being under neuroleptics scale and the subjective deficit syndrome scale. Psychopharmacology 162, 24-28.

Heinrichs D.W., Hanlon T.E. \& Carpenter W.T. (1984). The Quality of life scale: an instrument for rating the schizophrenic deficit syndrome. Schizophrenia Bulletin 10, 388-398.
Hogan T.P., Awad A.G. \& Eastwood M.R. (1983). A self-report scale predictive of drug compliance in schizophrenia: reliability and discriminate validity. Psychological Medicine 13, 177-183.

Karow A. \& Naber D. (2002). Subjective well-being and quality of life under atypical antipsychotic treatment. Psychopharmacology 162, 3-10.

Karow A., Czekalla J., Dittmann R.W., Schacht A., Wagner T., Lambert M., Schimmelmann B.G. \& Naber D. (2007). Association of subjective well-being, symptoms, and side effects with compliance after 12 months of treatment in schizophrenia. Journal of Clinical Psychiatry $68,75-80$.

Lambert M., Schimmelmann B.G., Naber D., Schacht A., Karow A., Wagner T. \& Czekalla J. (2006). Prediction of remission as a combination of symptomatic and functional remission and adequate subjective well-being in 2960 patients with schizophrenia. Journal of Clinical Psychiatry 67, 1690-1697.

Lambert M., Naber D., Eich F.X., Schacht A., Linden M. 6 Schimmelmann B.G. (2007). Remission of severely impaired subjective wellbeing in 727 patients with schizophrenia treated with amisulpride. Acta Psychiatrica Scandinavica 115, 106-113.

Meltzer H.Y., Burnett S., Bastani B. \& Ramirez L.F. (1990). Effects of six months of clozapine treatment on the quality of life of chronic schizophrenic patients. Hospital Community Psychiatry 41, 892-897.

Mizrahi R., Rusjan P., Agid O., Graff A., Mamo D.C., Zipursky R.B. \& Kapur S. (2007). Adverse subjective experience with antipsychotics and its relationship to striatal and extrastriatal D2 receptors: a PET study in schizophrenia. American Journal of Psychiatry 164, 630-637.

Naber D. (1995). A self-rating to measure subjective effects of neuroleptic drugs, relationships to objective psychopathology, quality of life and other clinical variables. International Clinical Psychopharmacology 10, Suppl. 3, 133-138.

Naber D. (2005). Subjective effects of antipsychotic treatment. Acta Psychiatrica Scandinavica 111, 81-83.

Naber D., Moritz S., Lambert M., Pajonk F.G., Holzbach R., Mass R. \& Andresen B. (2001). Improvement of schizophrenic patients' subjective well-being under atypical antipsychotic drugs. Schizophrenia Research 50, 79-88.

Naber D., Riedel M., Klimke A., Vorbach E.U., Lambert M., Kühn K.U., Bender S., Bandelow B., Lemmer W., Moritz S. \& Dittmann R.W. (2005). Randomized double blind comparison of olanzapine vs. clozapine on subjective well-being and clinical outcome in patients with schizophrenia. Acta Psychiatrica Scandinavica 111, 106-115.

Putzhammer A., Perfahl M., Pfeiff L. \& Hajak G. (2005). Correlation of subjective well-being in schizophrenic patients with gait parameters, expert-rated motor disturbances, and psychopathological status. Pharmacopsychiatry 38, 132-138.

van Putten T., May P.R.A. \& Marder S.R. (1981). Subjective response to antipsychotic drugs. Archives of General Psychiatry 38, 187-190.

Voruganti L. \& Awad A.G. (2004). Neuroleptic dysphoria: towards a new synthesis. Psychopharmacology 171, 121-132.

Voruganti K., Heslegrave R.J., Awad A.G. \& Seeman M.V. (1998). Quality of life measurement in schizophrenia: reconciling the quest for subjectivity with the question of reliability. Psychological Medicine 28, 165-172.

Voruganti L., Cortese L., Oyewumi L., Cernovsky Z., Zirul S. \& Awad A. (2000). Comparative evaluation of conventional and novel antipsychotic drugs with reference to their subjective tolerability, sideeffect profile and impact on quality of life. Schizophrenia Research 43, $135-145$.

Wehmeier P.M., Kluge M., Schneider E., Schacht A., Wagner T. \& Schreiber W. (2007a). Quality of life and subjective well-being during treatment with antipsychotics in out-patients with schizophrenia. Progress in Neuropsychopharmacology \& Biological Psychiatry 31, 703-712.

Wehmeier P.M., Kluge M., Schacht A., Helsberg K. \& Schreiber W. (2007b). Correlation of physician and patient rated quality of life during antipsychotic treatment in outpatients with schizophrenia. Schizophrenia Research 91, 178-186.

Windgassen K. (1992). Treatment with neuroleptics: the patient's perspective. Acta Psychiatrica Scandinavica 86, 405-410. 